\title{
Looking back to the future? Possible pandemic scenarios from the past with relevance for autumn/winter 2021, and the years ahead
}

\author{
Kaspar Staub 1,2,3* \\ ${ }^{1}$ Institute of Evolutionary Medicine, University of Zurich, Zurich, Switzerland \\ ${ }^{2}$ Institute of History, University of Bern, Switzerland \\ ${ }^{3}$ Swiss School of Public Health SSPH+, Zurich, Switzerland \\ * Corresponding author: kaspar.staub@iem.uzh.ch
}

\begin{abstract}
In many places the current coronavirus pandemic is the most severe pandemic since the 1918 influenza pandemic ("Spanish flu"). In many European countries before 2020, past experiences with pandemics had not been sufficiently studied and were no longer present in the minds of the general public or authorities. This article highlights scenarios from the past that may offer guidance as we move towards autumn and winter 2021. High quality morbidity data from the Swiss canton of Bern 1918-1930 is re-used here and complemented with similar data from 1957, SARS-CoV-2 data from 2020, as well as temperature series for all three years. A first possible scenario that emerges from experiences in all three pandemic years is that the onset of the fall waves at the beginning of October, occurred 0-2 weeks after the first drop in temperatures at the end of September (calendar week 39). This temporal coincidence can also be coincidental, and does not imply causality. However, this risk is also present for the coming autumn of 2021, all the more so if the case numbers will not be low everywhere then because of the delta variant. When temperatures start to fall, people will stay indoors more, which will increase the risk of infection for the unprotected or only partially protected subgroups of the population. In the winter of 1920, the influenza virus returned in the form of a relatively strong "echo" wave probably due to incomplete immunization of the population and/or virus mutations, and thereafter in the form of milder seasonal waves. This is a second scenario that many experts also consider possible for SARS-CoV-2. We do not know yet what will happen in autumn/winter 2021 and in the years to come. However, the past at least provides some scenarios of what happened in partly comparable situations in 1918 and thereafter. To not at least consider these possible scenarios in pandemic planning for the coming period would be a missed opportunity.
\end{abstract}


In July 2021, the world is still stuck in the coronavirus pandemic caused by the SARS-CoV-2 virus. It is now clear that in many places this is the most severe pandemic since the 1918 influenza pandemic ("Spanish flu"), ${ }^{1}$ and the impact on health and society is very large and ongoing. Now that most countries have been through several COVID-19 waves, the question is what challenges we might face in the second half of the year 2021 and in the years ahead?

When a society is confronted with a novel pathogen for which there is little or no immunity, as was the case in 1918 with the H1N1 influenza A virus, and in 2020 with the SARS-CoV-2 virus, lessons from the past can help inform approaches to the new challenge and the current time context. ${ }^{2,3}$ However, in spring 2020 it quickly became clear that in many European countries past experiences with pandemics had not been sufficiently well studied and were no longer present in the minds of the general public and authorities. ${ }^{4}$ This is not least due to the fact that many of these countries had been spared by the devastating effects of pandemics since the first half of the 20th century. Unfortunately, it has been shown that certain mistakes made in 1918 were repeated in 2020, especially in the management of subsequent waves, ${ }^{5}$ and thus that it can be fatal to ignore the lessons of the past. ${ }^{6}$

This article highlights scenarios from the past that may offer guidance as we move towards the end of the acute phase of the coronavirus pandemic. It uses the quantitative data of the 1918 influenza pandemic in the Swiss canton of Bern, which was recently analyzed in detail. ${ }^{5}$ Here, these published data of physician-reported new cases of influenza-like illnesses (ILI) are re-used for the period between July 1918 and December 1930, and newly enhanced by the years 1957-1958. ${ }^{7}$ Newly reported and laboratory-confirmed cases of Sars-CoV-2 infections for the canton of Bern for the calendar weeks between March 2020 and June 2021 were extracted from the publicly available data of the cantonal authorities. Temperature data (daily maximum) from MeteoSwiss/IDAWEB for the Bern (Zollikofen) weather station were averaged for calendar weeks. While the data only covers a small geographical area, it is nevertheless of international relevance as the challenges it addresses might be of relevance to now and for many other places.

A first possible scenario - shown here for the first time - that emerges from experiences in 1918, 1957, and 2020 is that in all three pandemics, the onset of the fall wave in October occurred 0-2 weeks after the first drop in temperatures in calendar week 39 at the end of September (Figure 1A-C). In 1918 and 2020, all restrictions had been completely lifted after the first wave, and the number of reported new cases was at a comparatively stable and low level in September. In 1957, the first cases of influenza (H2N2 influenza A virus) were reported in Switzerland at the beginning of September, after which the number of newly reported cases initially remained relatively low. In all three pandemic years, the weekly averaged daily maximum temperatures started to fall below 17 degrees Celsius in calendar week 39, and this was followed by a noticeable increase in newly reported infections. In the 1918 and 1957 influenza pandemics this was the case already 0-1 weeks later, in 2020 with the coronavirus it took 1-2 weeks and thus somewhat longer for case numbers to rise.

This slight difference makes sense as the incubation period for SARS-CoV-2 is longer than for influenza in 1918 and 1957, and it also takes longer for a COVID-19 case to be reported to the authorities due to laboratory testing. It is very important to note that the coincidence of pandemic growth with a temperature drop could be due to chance and is presented purely descriptively, and does not necessarily imply causality. It is also clear that the emergence of new pandemic waves can only be explained multi-factorially and not by temperature curves alone. On the other hand, this correlation is likely not spurious: it has now widely been recognized that staying indoors significantly increases the risk of transmission via droplets and aerosols. ${ }^{8}$ When temperatures started to fall at the end of September and beginning of October in 1918, 1957 and 2020, people started to stay indoors more, which increased the risk of transmission.

Will history repeat itself (again) at the end of September 2021? Not necessarily. The majority of the population will be at least partially protected by having been previously infected and or vaccinated, although the strength of this protection will be influenced by the circulating virus variant(s). However, another part of the population (and among them many young children) will still 
be unprotected or only partially protected, and for these population groups, the risk of infection will again increase considerably as life moves primarily indoors at the end of September. This also applies if there is a new wave in the course of the summer, driven for example by the delta variant. Then week 39 poses an even higher risk because the number of cases will not be low when life moves indoor again.

A second scenario that the canton of Bern data prompts us to consider is the occurrence of echo waves. In January of 1920 there followed a later wave, and in the years thereafter the influenza virus returned every 1-2 years in weaker seasonal waves (Figure 1D). The echo wave in the winter of 1920 was noticeable beyond Switzerland, ${ }^{9}$ and was probably partly due to incomplete immunization of the population, ${ }^{10}$ and possibly also due to virus mutations. ${ }^{11}$ That the influenza virus returned after the $1918 / 1919$ pandemic in a strongly winter-seasonal form with an annual or biennial periodicity is known from other European countries, ${ }^{12}$ but there is also evidence that there were sporadic influenza waves even before 1918. ${ }^{13}$ Will SARS-CoV-2 also return in the winter of 2021 in the form of a relatively strong echo wave due to incomplete immunization, and thereafter in the form of milder seasonal waves? This is at least one scenario that many experts consider possible.

We don't know yet what will happen in autumn/winter 2021 and years to come. Despite obvious parallels, there are still important differences between past and the present pandemic. But the past at least provides some scenarios of what happened in partly comparable situations in 1918 and the years thereafter. To not at least consider these possible scenarios in pandemic planning for the coming period would be a missed opportunity. When life shifts indoors again due to temperature, this increases the risk of infection, as these three pandemic examples from the past show. This is all the more true if a virus variant is rampant that is more contagious than the previous variants, and if the infection numbers in September 2021 will not be low everywhere. Which scenarios will or will not occur also depends on how many people are at least partially protected by a previous infection and/or the level of vaccination uptake. Thanks to biomedical progress, highly effective vaccines have been developed in record time, and virus evolution can be monitored virtually in real time today. Let us make use of this historically incomparable advantage! 


\section{Bibliography}

1 Morens DM, Taubenberger JK, Fauci AS. A Centenary Tale of Two Pandemics: The 1918 Influenza Pandemic and COVID-19, Part I. Am J Public Health 2021; 111: 1086-94.

2 Morens DM, Daszak P, Taubenberger JK. Escaping Pandora's Box - Another Novel Coronavirus. N Engl J Med 2020; 382: 1293-5.

3 Giles-Vernick T. Influenza and public health: learning from past pandemics. London: Earthscan, 2010.

4 Staub K, Rühli F, Floris J. The "Pandemic Gap" in Switzerland across the 20th century, and the necessity of increased science communication of past pandemic experiences. Swiss Med. Wkly. 2020. https://smw.ch/op-eds/post/the-pandemic-gap.

5 Staub K, Jüni P, Urner M, et al. Public Health Interventions, Epidemic Growth, and Regional Variation of the 1918 Influenza Pandemic Outbreak in a Swiss Canton and Its Greater Regions. Ann Intern Med 2021; 174: 533-9.

6 Mooney G. The Dangers of Ignoring History Lessons During a Pandemic. Ann Intern Med 2021; 174: 556-7.

7 Schweiz Bundesamt für Gesundheitswesen. Bulletin des Eidgenössischen Gesundheitsamtes, 1920-1979. Bern: Hallwag.

8 Greenhalgh T, Jimenez JL, Prather KA, Tufekci Z, Fisman D, Schooley R. Ten scientific reasons in support of airborne transmission of SARS-CoV-2. Lancet 2021; 397: 1603-5.

9 Ansart S, Pelat C, Boelle P-Y, Carrat F, Flahault A, Valleron A-J. Mortality burden of the 1918-1919 influenza pandemic in Europe. Influenza Other Respi Viruses 2009; 3: 99-106.

10 Bogaert K. Cross protection between the first and second waves of the 1918 influenza pandemic among soldiers of the Canadian Expeditionary Force (CEF) in Ontario. Vaccine 2015; 33: 7232-8.

11 Patrono LV, Vrancken B, Budt M, et al. Archival influenza virus genomes from Europe reveal genomic and phenotypic variability during the 1918 pandemic. bioRxiv 2021; : 2021.05.14.444134.

12 Weinberger DM, Krause TG, Molbak K, et al. Influenza Epidemics in Iceland Over 9 Decades: Changes in Timing and Synchrony With the United States and Europe. Am J Epidemiol 2012; 176: 649-55.

13 Schmid F. Die Influenza in Der Schweiz in den Jahren 1889-1894. Bern, 1895. 


\section{Author statements}

\section{Acknowledgements:}

The author thanks Peter Jüni, Martin Urner, Marcel Zwahlen, Milo Puhan, Olivia Keiser, Joël Floris, Katarina Matthes, and Frank Rühli for helpful comments and support.

\section{Funding:}

Foundation for Research in Science and the Humanities at the University of Zurich (grant STWF-21011); Mäxi Foundation, Zürich

Competing interests: The author declares no competing interests.

Data and materials availability: All data used in this article is provided as online Supplementary Material. 
Figure 1: Weekly totals of newly reported cases per 100'000 inhabitants of influenza-like-illness (ILI) in 1918/1919 (A $\left.\mathbf{A}^{5}\right)$ and 1957/1958 (B), and laboratory-confirmed SARS-CoV-2 infections in 2020/2021 (C) in the Canton of Bern (BE), plotted against weekly averages of daily maximum temperatures in the City of Bern (red lines, dotted reference lines indicates $17^{\circ} \mathrm{C}$ ). a) Reporting obligation introduced; b) Complete lifting of all measures after first wave; c) Reporting obligation introduced; d) Change of testing strategy: All people with symptoms should get tested; e) Complete lifting of all measures after first wave. Blue shaded area: Weeks 39-41. D) Weekly totals of physicianreported new cases of influenza-like-illness (ILI) in the canton of Bern, 1918-1930. ${ }^{5}$

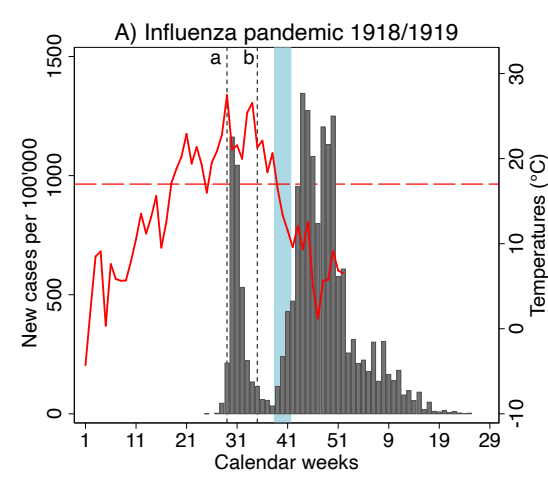

New cases BE — Temperatures Bern

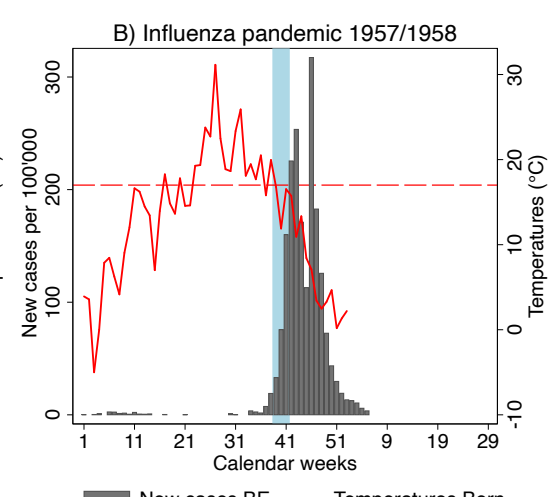

New cases BE — Temperatures Bern

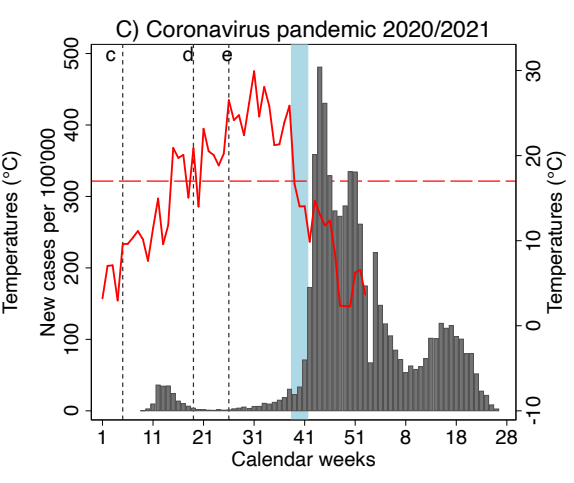

New cases BE — Temperatures Bern

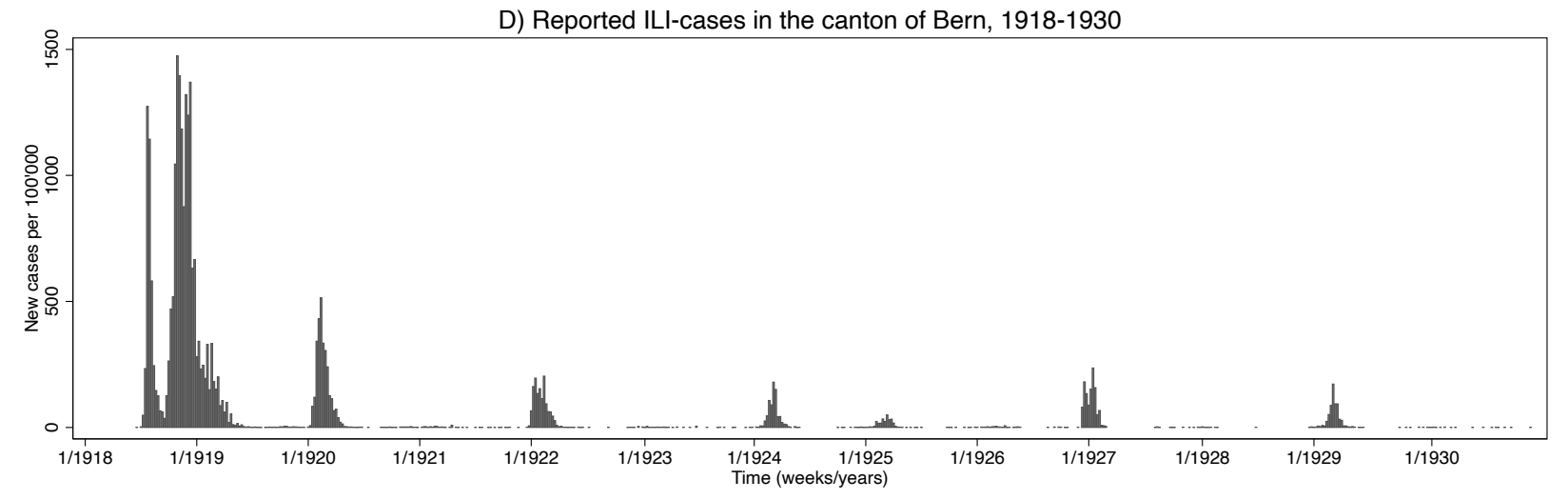

